\title{
Chemoselective Radical Cleavage of Cbz-Protected Nitrogen Compounds
}

\author{
M.-Lluïsa Bennasar*, Tomàs Roca, and Ariadna Padullés \\ Laboratory of Organic Chemistry, Faculty of Pharmacy, University of Barcelona, Barcelona \\ 08028, Spain
}

\section{Supporting Information}

$\mathrm{N}$-Cbz derivatives 4, 8-12 and 14-16 were prepared from commercially available azoles, amides or amines by treatment with $\mathrm{Cbz}-\mathrm{Cl}$, under standard basic conditions. For 4, 9 and 12: $\mathrm{NaH}$, THF; for 8 and 11: $\mathrm{Et}_{3} \mathrm{~N}$, THF; for 10, 15 and $16(\mathrm{KOH}$, acetone).

The corresponding deprotected products were compared with authentic samples and their data were in full agreement.

Methyl 4-[1-(benzyloxycarbonyl)-3-indolyl]-4-oxobutanoate (2).

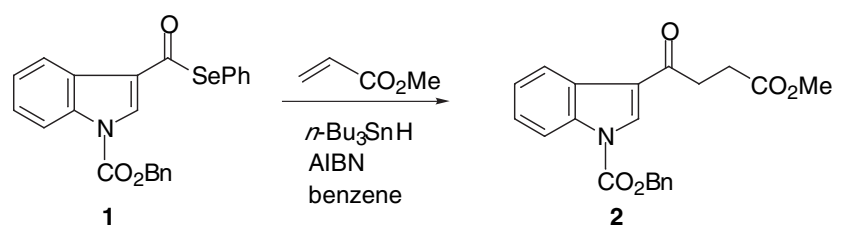

$n$ - $\mathrm{Bu}_{3} \mathrm{SnH}(0.21 \mathrm{~mL}, 0.8 \mathrm{mmol})$ in $\mathrm{C}_{6} \mathrm{H}_{6}(3 \mathrm{~mL})$ was added over a period of $1 \mathrm{~h}$ (syringe pump) to a heated (reflux) solution of $1(0.28 \mathrm{~g}, 0.64 \mathrm{mmol})$, methyl acrylate $(0.27 \mathrm{~g}, 3.2 \mathrm{mmol})$ and AIBN (10 mg, $0.06 \mathrm{mmol}$ ) in $\mathrm{C}_{6} \mathrm{H}_{6}(6 \mathrm{~mL})$. After additional 2-3 $\mathrm{h}$ at reflux, the solution was concentrated under reduced pressure, and the residue was partitioned between hexanes $(5 \mathrm{~mL})$ and acetonitrile $(5 \mathrm{~mL})$, and the polar layer was washed with hexanes $(3 \times 5 \mathrm{~mL})$ to remove tin compounds. The solvent was removed and the crude product was chromatographed $\left(\mathrm{SiO}_{2}\right.$, flash, 75:25 hexanes-AcOEt) to give 2: $0.11 \mathrm{~g}(47 \%) ;{ }^{1} \mathrm{H} \mathrm{NMR}\left(\mathrm{CDCl}_{3}, 300 \mathrm{MHz}\right) 2.78(\mathrm{t}, \mathrm{J}=6.8 \mathrm{~Hz}$, $2 \mathrm{H}), 3.24(\mathrm{t}, J=6.8 \mathrm{~Hz}, 2 \mathrm{H}), 3.70(\mathrm{~s}, 3 \mathrm{H}), 5.50(\mathrm{~s}, 2 \mathrm{H}), 7.43(\mathrm{~m}, 7 \mathrm{H}), 8.20(\mathrm{~m}, 1 \mathrm{H}), 8.30(\mathrm{~m}$, $1 \mathrm{H}), 8.32(\mathrm{~s}, 1 \mathrm{H})$; HRMS calcd for $\mathrm{C}_{21} \mathrm{H}_{19} \mathrm{NO}_{5} 365.1257$, found 365.1250 .

Representative Deprotection Procedure: Trans-3-(benzyloxycarbonyl)-4-(1-methyl-3indolylcarbonyl)-2-piperidone.

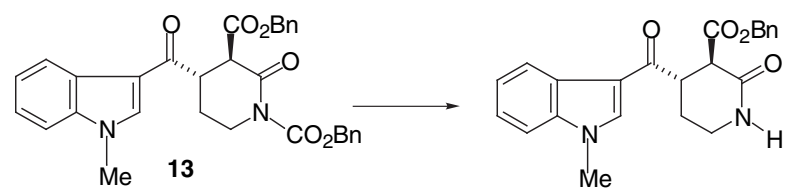

A solution of 2-piperidone $\mathbf{1 3}^{1}(210 \mathrm{mg}, 0.4 \mathrm{mmol}), n-\mathrm{Bu}_{3} \mathrm{SnH}(0.16 \mathrm{~mL}, 0.6 \mathrm{mmol})$, and AIBN (7 mg, $0.04 \mathrm{mmol})$ in ethylbenzene $(4 \mathrm{~mL})$ was heated at reflux temperature. After the mixture was stirred for $0.5 \mathrm{~h}$, a second portion of AIBN $(7 \mathrm{mg}, 0.04 \mathrm{mmol})$ was added. The mixture was stirred at reflux temperature for an additional $0.5 \mathrm{~h}$ and then concentrated under reduced pressure. The residue was partitioned between hexanes $(10 \mathrm{~mL})$ and acetonitrile $(10 \mathrm{~mL})$, and the polar layer was washed with hexanes $(3 \times 10 \mathrm{~mL})$ to remove tin compounds. The solvent was removed, and the crude product was chromatographed $\left(\mathrm{SiO}_{2}\right.$, flash, 99:1 AcOEt-MeOH) to give the secondary amide: $140 \mathrm{mg}(90 \%) ;{ }^{1} \mathrm{H} \mathrm{NMR}\left(\mathrm{CDCl}_{3}, 300 \mathrm{MHz},\right) 2.13(\mathrm{~m}, 2 \mathrm{H}), 3.44(\mathrm{~m}$, 2H), $3.68(\mathrm{~s}, 3 \mathrm{H}), 3.92(\mathrm{dt}, J=4.4,9.6,10 \mathrm{~Hz}, 1 \mathrm{H}), 4.03(\mathrm{~d}, J=10.2 \mathrm{~Hz}, 1 \mathrm{H}), 5.13(\mathrm{~s}, 2 \mathrm{H}), 6.57$ (br s, 1H), 7.10-7.40 (m, 8H), $7.74(\mathrm{~s}, 1 \mathrm{H}), 8.38(\mathrm{~m}, 1 \mathrm{H}) ;{ }^{13} \mathrm{C} \mathrm{NMR}\left(\mathrm{CDCl}_{3}, 50.3 \mathrm{MHz}\right) 26.1$ $\left(\mathrm{CH}_{2}\right), 33.6\left(\mathrm{CH}_{3}\right), 41.0\left(\mathrm{CH}_{2}\right), 44.6(\mathrm{CH}), 50.6(\mathrm{CH}), 67.1\left(\mathrm{CH}_{2}\right), 109.7(\mathrm{CH}), 114.7(\mathrm{C}), 122.6$ $(\mathrm{CH}), 122.9(\mathrm{CH}), 123.7(\mathrm{CH}), 126.4(\mathrm{C}), 127.6(\mathrm{CH}), 127.7(\mathrm{CH}), 128.1(\mathrm{CH}), 135.5(\mathrm{C})$, 
$136.2(\mathrm{CH}), 137.6(\mathrm{C}), 168.1(\mathrm{C}), 170.1(\mathrm{C}), 193.3(\mathrm{C})$. Anal. Caled for $\mathrm{C}_{23} \mathrm{H}_{22} \mathrm{~N}_{2} \mathrm{O}_{4}$ : C, 70.71; H, 5.67; N, 7.17.Found: C, 70.64; H, 5.86; N, 7.06.

${ }^{1}$ Bennasar, M.-L.; Roca, T.; Griera, R.; Bosch, J. J. Org. Chem. 2002, 67, 6268-6271.

Methyl (3-indolyl)-4-oxobutanoate (3).

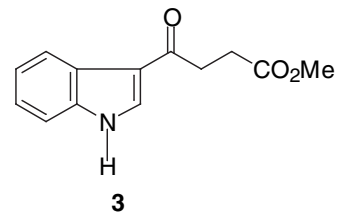

Prepared as above from 2: 95\%; ${ }^{1} \mathrm{H}$ NMR $\left(\mathrm{CDCl}_{3}, 400 \mathrm{MHz}\right) 2.81(\mathrm{t}, J=6.8 \mathrm{~Hz}, 2 \mathrm{H}), 3.25(\mathrm{t}, J$ $=6.8 \mathrm{~Hz}, 2 \mathrm{H}), 3.71(\mathrm{~s}, 3 \mathrm{H}), 7.28(\mathrm{~m}, 2 \mathrm{H}), 7.41(\mathrm{~m}, 1 \mathrm{H}), 7.93(\mathrm{~d}, \mathrm{~J}=3.2 \mathrm{~Hz}), 8.37(\mathrm{~m}, 1 \mathrm{H}), 8.65$ (br s, 1H); ${ }^{13} \mathrm{C} \mathrm{NMR}\left(\mathrm{CDCl}_{3}, 50.3 \mathrm{MHz}\right) 28.2\left(\mathrm{CH}_{2}\right), 34.3\left(\mathrm{CH}_{2}\right), 51.8\left(\mathrm{CH}_{3}\right), 111.3(\mathrm{CH}), 117.8$ (C), $122.4(\mathrm{CH}), 122.7(\mathrm{CH}), 123.8(\mathrm{CH}), 125.4(\mathrm{C}), 130.9(\mathrm{CH}), 136.2(\mathrm{C}), 173.8(\mathrm{C}), 193.5$ (C); HRMS calcd for $\mathrm{C}_{13} \mathrm{H}_{13} \mathrm{NO}_{3} 231.0894$, found 231.0899. 